\title{
Microcontroller-based System for Voltage Monitoring, Protection and Recovery using Proteus VSM Software
}

\author{
Mohammed Y.M. Alnaham \\ Graduate student \\ Sudan University of Science and Technology \\ Khartoum, Sudan
}

\author{
Mamoun M.A. Suliman, Ph.D \\ Associate Professor \\ Sudan University of Science and Technology \\ Khartoum, Sudan
}

\begin{abstract}
To protect electric and electronic device against voltage drop, overshoot, and fluctuation of the main supply, a microcontroller-based circuit is designed. The circuit will keep monitoring the power supply and ensure the main voltage connected to the board feeding the controlled devices connected if it measures within a required voltage range (RVR). If the voltage falls below or above the RVR then the main supply would be disconnected from the feeding board. The circuit then will keep monitoring the voltage and reconnect it to the feeding board once its amplitude falls back stable in the required voltage range. The design was simulated successfully using Proteus software.
\end{abstract}

\section{Keywords}

Microcontroller, Proteus, Monitoring, Protection, Recovery,

\section{INTRODUCTION}

All countries suffer from grid voltage instability at above or under voltage standards that cause home or office electric appliances damage, like computers, refrigerators, TV sets, or other equipment[1]. This paper aims for the design of an embedded system using a microcontroller to monitor the amplitude of the main voltage and interrupts the supply when it increases or decreases from a programmed range. Furthermore, the system will enable the recovery of the supply if already disconnected, when the voltage assumes again the required range. The microcontroller is a specialized microprocessor that houses much of the support circuitry onboard, such as ROM, RAM, serial communications ports, ADC, etc [2]. Microcontrollers have become the heart of the new technologies that are being introduced daily. A microcontroller is mainly a single chip microprocessor suited for control and automation of machines and processes. Today, microcontrollers are used in many disciplines of life for carrying out automated tasks in a more accurate manner [3-4]. The block diagram of the microcontroller system is shown in Fig 1.

Microcontroller essentially consists of Central Processing Unit (CPU), timers and counters, interrupts, memory, input/output ports, analog to digital converters (ADC) on a single chip [5]. With this single chip integrated circuit design of the microcontroller the size of control board is reduced and power consumption is low [6].

This paper presents the design and simulation of the microcontroller based system for voltage monitoring, protection, and recovery using PIC877A microcontroller simulated on Proteus VSM Software.

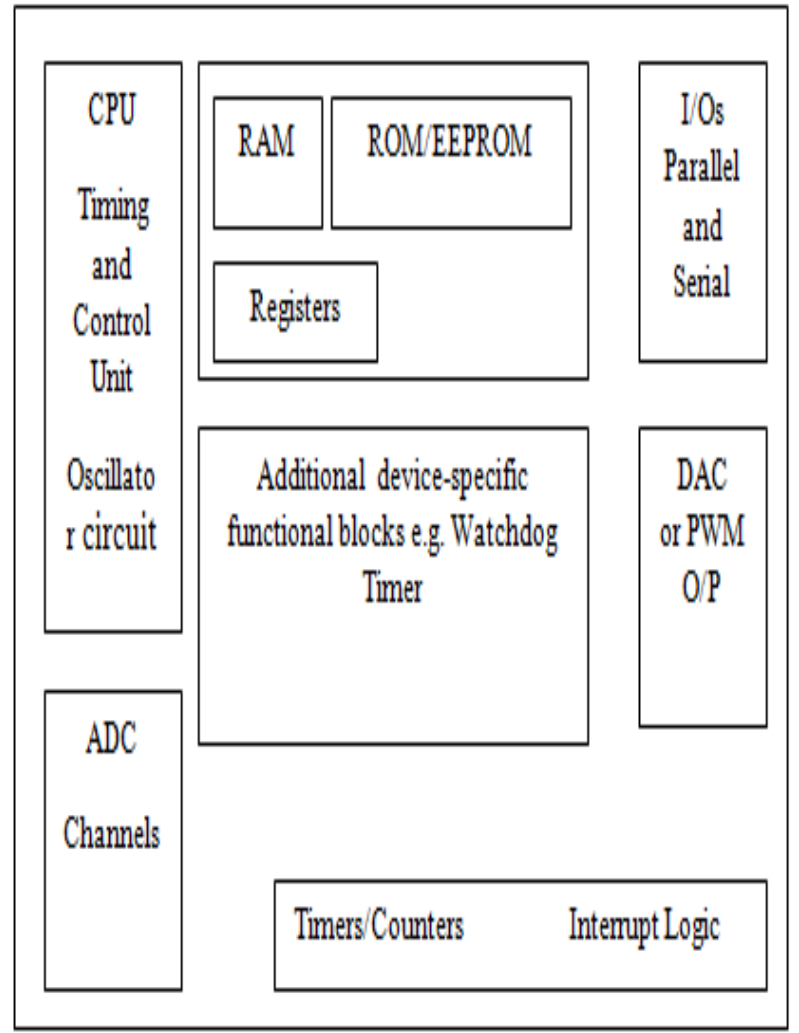

Fig 1: Microcontroller System

\section{PROTEUS VSM SOFWARE}

Proteus software is from British Labcenter Electronics Company. It is widely used globally. Besides the function other EDA tools have, like principle layout, PCB automatic or artificial wiring and the SPICE simulation, its revolution feature is circuit simulation's interactive [7].

Proteus Virtual System Modeling (VSM) combines mixed mode SPICE circuit simulation, animated components and microprocessor models to facilitate co-simulation of complete microcontroller based designs. For the first time ever, it is possible to develop and test such designs before a physical prototype is constructed [8]

Proteus simulation environment includes ISIS and ARES. In the ISIS environment, electronic circuit can be directly stimulated in the stage of schematic. It also provides virtual machines, simulation of the input signal which makes a convenient simulation. ARES is mainly used for PCB (Printed Circuit Board-PCB) design. The device library provides schematic device and its package. It can design printed circuit board in this environment [9]. 


\section{DEVELOPMENT TOOLS and EXPERIMENTAL RESULTS \\ 3.1 System Design}

System is constructed and implemented as shown in Fig 2.



Fig 2: Block diagram of voltage controller

The main component is PIC16F877A MCU that senses the received voltage and compares it with reference value fed from MCU (PORTC). AC voltage source (220V) is applied to $9 \mathrm{~V}$ step-down transformer that transforms the 220 volt to 9 volt, then apply it to 8 bits ADC to convert to 8 bits digital; this 8 bits binary word is compared with 8 bits reference value coming from PORTC of PIC 16F877A MCU. If the 8 bits generated from $\mathrm{ADC}$ is greater than the 8 bits representing maximum-voltage reference, that means the voltage is greater than 230 volt, and must be disconnected. If the 8 bits from $\mathrm{ADC}$ is less than the 8 bits low-voltage reference, that means the voltage is less than 215 volts, and must be disconnected. If the 8 bits that come from $\mathrm{ADC}$ is equal to the 8 bits which come from MCUs PORTC, that means the voltage is in normal range and one minute delay must be applied to insure the stability of voltage within the required range. After one minute in which the voltage remains in range, the voltage source will be connected to device. The warning message block is constructed of three, LEDs. Red LED which indicates high voltage, yellow LED indicates low voltage, and green LED indicating normal voltage. The relay will respond to the proper signal from the microcontroller to switch the voltage supply on and off. Input reference RVR block indicates users can adjust reference voltage to any range. External battery is necessary for microcontroller operating in case of supply voltage source interruption. Digital comparator CD4063BMS is responsible for comparing sensed voltage with reference. The transistor $2 \mathrm{~N} 1893$ is to sense the output decision signal that comes from $\mathrm{MCU}$, and trigger the relay to connect or disconnect the $\mathrm{AC}$ voltage.
The circuit diagram of the design is shown in Fig 3.

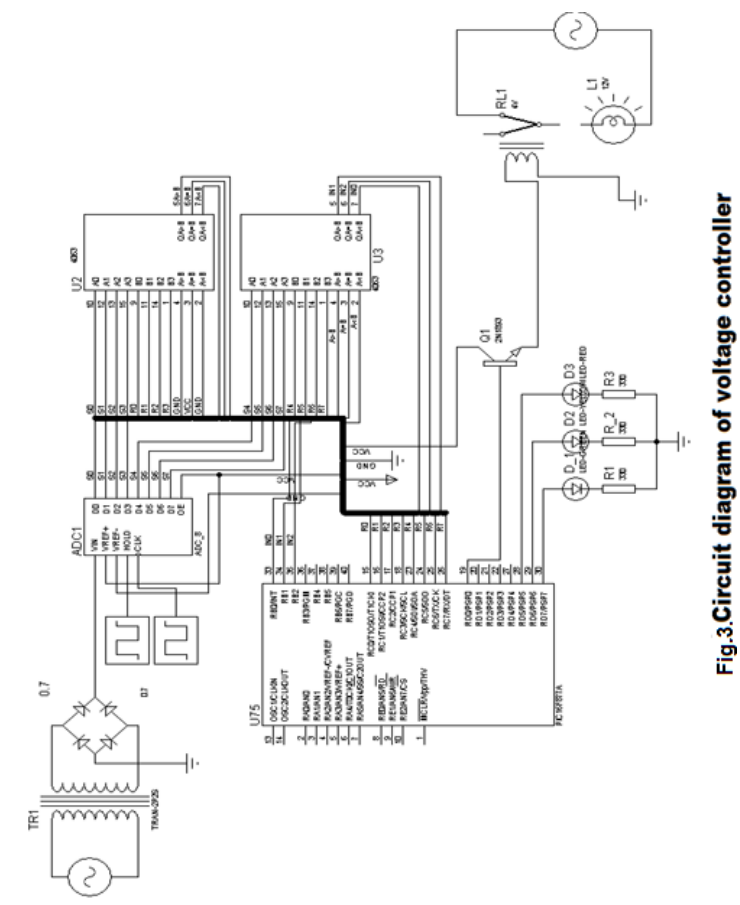

\subsection{Flowchart of the System}

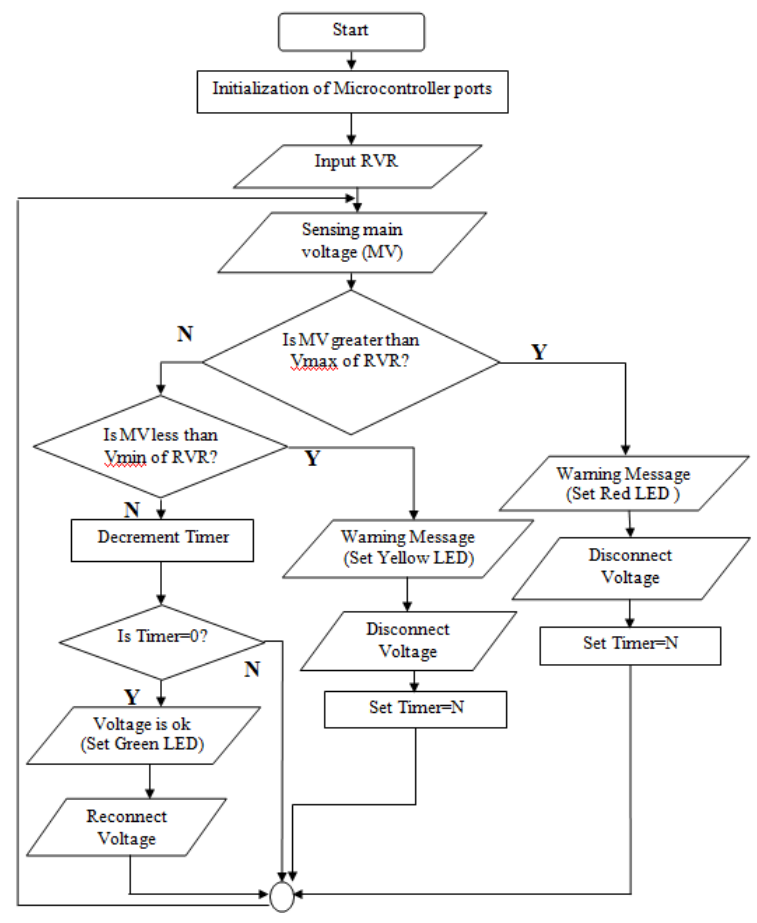

Fig.4.Flowchart of the system

\subsection{Program Code of System}

To make it easy to understand, the program code has been sectionalized into three parts:

- Sensor code

- Processing code

- Wait cycle code

First, part of code that is responsible of sensing the magnitude of source voltage is illustrated in Fig 5 . 


sensol;
MOVLW OXE2;:Move OXE2 to work register
MOWWF PORTC; Send the OXE2 from work register to comparator as reference
BTFSS PORTB, 1; Test the main votage state to Know is it very high (Greater than 230.5 volts)
GOTO TEST1; Go to test if voltage is not high
GOTO HV; Go to subroutin HV (high voltage)
TEST1
MOVLW OXDO;Move OXDO to work register
MOWWF PORTC; Send the OXDO to comparator as reference
BTFSS PORTB, O; Test the main votage state to know is it very low (less than 214.5 volt)
GOTO NV; Go to subroutin NV (normal voltage )
GOTO LV; Go to subroutin LV (low voltage)

Fig 5: Sensor Code Part

As shown in Figure 5, first MOVLW OXE2 instruction and MOVWF PORTC feed value to a comparator from PORTC of microcontroller as reference to compare with output of ADC (sensed value). If the sensed voltage is greater than 0XE2 that means the voltage is greater than RVR and must be switched off by relay; if the voltage is less than 0XE2 check if the voltage is lower than RVR by comparing the sensed voltage with OXD0. If the voltage is less than OXD0 it means that the voltage is less than RVR and must be switched off by relay; otherwise, the voltage is in RVR range and it will not be interrupted.

Second, part of code responsible for control and action is illustrated in Fig 6:

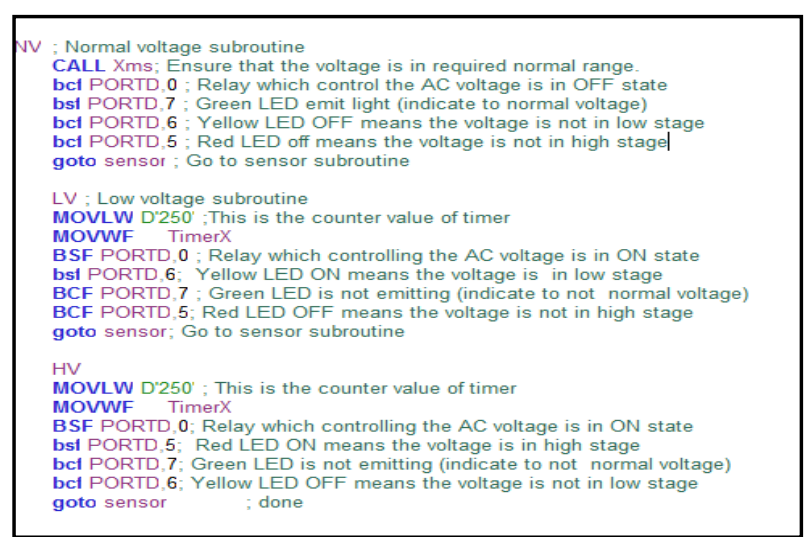

Fig 6: Control code part

As shown in Fig 6, if the voltage is within RVR, send 0 signal to relay, and 1 signal to green LED. If the voltage is greater than RVR, send 1 signal to relay and 1 signal to red LED; if the voltage is less than RVR send 1 signal to relay, and 1 signal to yellow LED.

Third, part of code subroutine that represents wait cycle (to ensure stability of voltage) is:

; 1ms delay with 1us cycle time (1000 cycles)

Onems

MOVLW D'250' ; Count for 1ms delay

MOVWF Timer1 ; Load count

Loop1 MOVLW 0XE2;Move 0XE2 to work register

MOVWF PORTC; Send the OXE2 from work register to comparator as reference
BTFSC PORTB, 1; Check if voltage is high

GOTO HV; Go to HV subroutine

DECFSZ Timer1 ; Count

GOTO Loop1 ; until Z

RETURN ; and finish

; Delay $\mathrm{Xms}, \mathrm{X}$ received in $\mathrm{W}$

Yms

MOVLW D'45'

MOVWF TimerY ; Count for $X$ ms

LoopY MOVLW 0XD0;Move 0XD0 to work register

MOVWF PORTC; Send the OXD0 to comparator as reference

CALL Onems ; Delay $1 \mathrm{~ms}$

BTFSC PORTB,0; Check if voltage is high

GOTO LV; Go to LV subroutine

DECFSZ TimerY ; Repeat $X$ times

GOTO LoopY ; until Z

RETURN ; and finish

Xms

MOVLW D'250'

MOVWF TimerX ; Count for X ms

LoopX

CALL Yms ; Delay $1 \mathrm{~ms}$

DECFSZ TimerX ; Repeat $X$ times

GOTO LoopX ; until Z

RETURN ; and finish

\subsection{Compile Program and Get Hexadecimal File}

Once code is written the next step is to get hexadecimal file. First, do MAKE to check the program doesn't contain any errors, and see the successful message to indicate that; after that, do BUILD ALL to get hexadecimal file, see Fig 7.

The source code is assembled (converted into machine code) by the assembler program MPASM, which creates the list of binary instruction codes. It is normally displayed as hexadecimal numbers and saved as progname hex.

Proteus simulation environment includes ISIS and ARES. In the ISIS environment, electronic circuit can be directly stimulated in the stage of schematic. It also provides virtual

machine, simulation of the input signal. ARES is mainly used for PCB design. The device library provides schematic tools and its package. It can design printed circuit board in this environment.

To program chip in Proteus, see Figure 8 hex code is downloaded to the PIC 16F877A chip. To download hex code to PIC chip there are four steps:

1- Double click on MCU

2- Edit component window will appear as shown in Fig 8 click on program file.

3- Choose the hex file from specific directory folder and double click on it.

4- Click ok , now the microcontroller is programmed. 


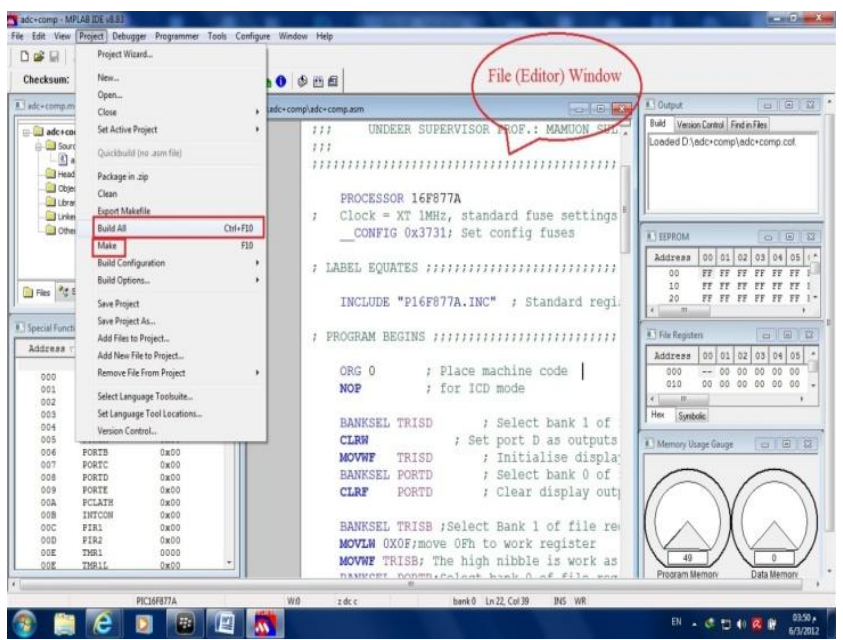

Fig 7: File (Editor ) window

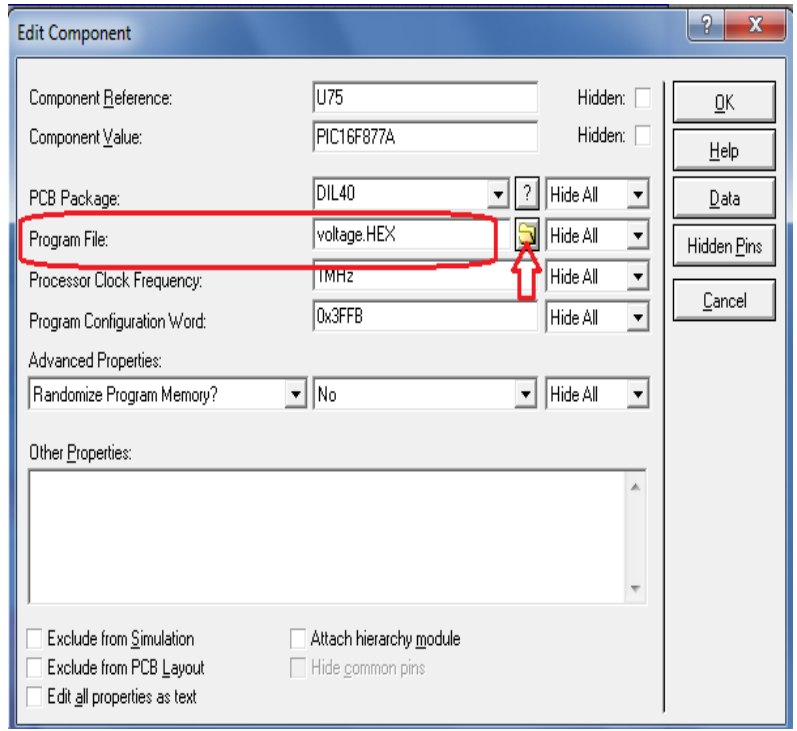

Fig 8: Edit component window

\subsection{Simulation And Experimental Results}

This part discusses the results of software environment (Proteus simulator). Normal voltage or Required Voltage Range RVR proposed here is in range of 215-230 (i.e. greater than low voltage (215 volt) and less than upper voltage (230 volt).

The result of software part (Proteus environment) is illustrated in Figures 9-11.

As shown in Fig 9, when the voltage is lower than RVR, the blue LED which represents the voltage supply is off, and yellow LED is lighted (indicating voltage is below normal range).

As shown in Fig 10, this shows the voltage within RVR, and the blue LED represents the connected voltage supply.

Green LED illustrates that the voltage is normal within RVR.

As shown in Fig 11 when the voltage is higher than RVR, the blue LED representing switched-off voltage supply (being disconnected), and red LED is lighted.

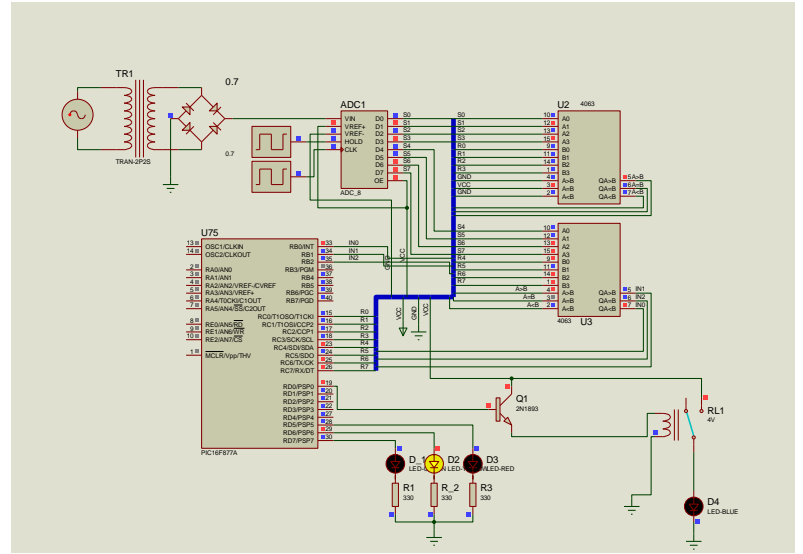

Fig 9: Low voltage illustration

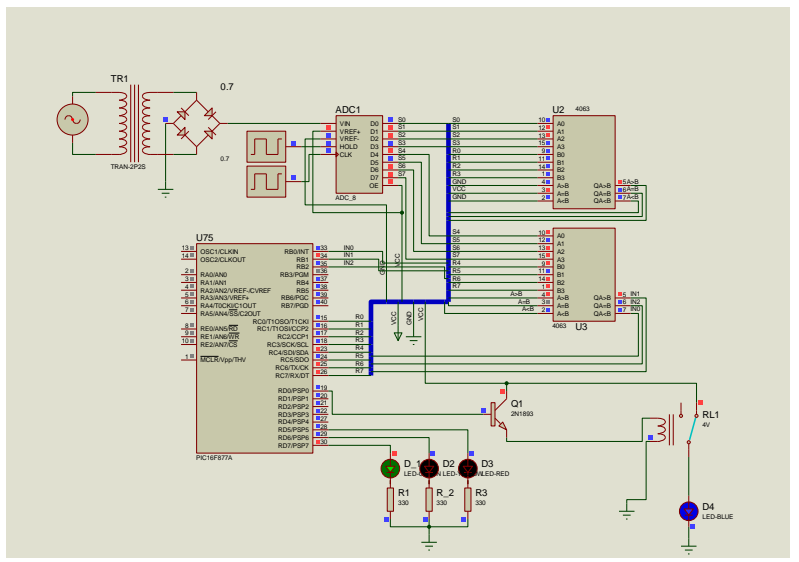

Fig 10: Normal voltage illustration

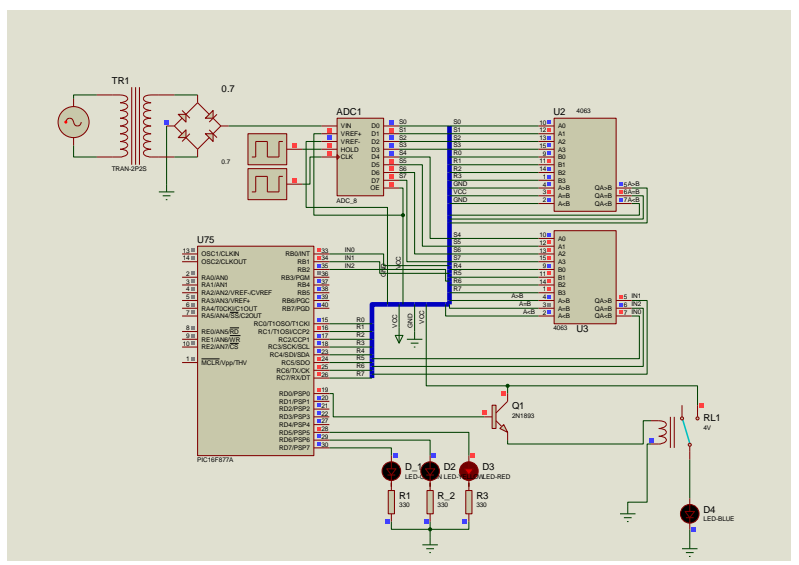

Fig 11: High voltage illustration

\section{CONCLUSION}

A microcontroller-circuit is designed to monitor the main voltage supply, and interrupt it if it falls below or above a required voltage range (RVR). If the voltage is disconnected, the microcontroller circuit will keep monitoring the voltage and only reconnect it back if it complies with the RVR and becomes stable. The simulation of the design was successful. The design is suitable for domestic use, given the fluctuations in main voltage supply worldwide, especially during summer and severe weather condition, and subsequent power-cuts. Voltage fluctuation leads to damage of electronic and electrical equipment connected to the main supply. 
This simulation used a range of RVR of 215- 230 volt as boundary of normal voltage; a keypad is recommended in the design to input the RVR by user. Keypad gives the advantage of picking any voltage range ( $110 \mathrm{~V}$ supply for example). Embedded system technique, which based on microcontroller is intelligent system which in case of use it as voltage monitoring to protect home or office electric appliances will provide more and more of valuable devices so this technique is favorable and useful.

\section{REFERENCES}

[1] Mohammed Y.M Alnaham. June 2012. "Microcontroller based System for Voltage Monitoring, Protection and Recovery", Master thesis, Under Supervisor Associate Professor Mamoun M.A Suliman. Sudan University of Science and Technology Khartoum, Sudan

[2] Paul Scherz [2000]," Practical Electronics for Inventors", The McGraw-Hill Companies, ISBN-13: 9780070580787, 1st Ed, April 15, 2000.

[3] T.-H Hsu, J.-F. Liu, P.-N Yu, W.-S. Lee, and J.-S. Hsu, "Development of an automatic parking system for vehicle," in Proc. of the International Conference on the IEEE Vehicle Power and Propulsion, Sept. 3-5 Harbin, China, 2008, pp. 1-6. .

[4] Md. M. Islam, F. H. Md. Rafi, A. F. Mitul and M. Ahmad, "Development of a Noninvasive Continuous Blood pressure Measurement and Monitoring system",
Proceedings of the International conference on ICIEV, May- 2012,pp. 1085-1090.

[5] Xiaodong Xia, Based on Single Chip Microcomputer Remote Wireless Control System Design. Coal Mine Machinery, vol. 32 (8), 2011, pp. 202-204.

[6] T. R. F Fulford-Jones, W. Gu-Yeon, and M Welsh, "A portable, low power, wireless two-lead EKG system," Proceedings of the 26th Annual International Conference of the IEEE Engineering in Medicine and Biology Society (EMBC-04), 1-5 Sept. 2004 , pp.2141-2144.

[7] Xu Xiumei; Pan Jinfeng; , "The simulation of temperature and humidity control system based on PROTEUS," Mechatronic Science, Electric Engineering and Computer (MEC), 2011 International Conference on Jilln, China , vol., no., pp.1896-1898, 19-22 Aug. 2011 doi:10.1109/MEC.2011.6025856

URL:http://ieeexplore.iee.org/stamp/stamp.jsp?tp=\&arn umber $=6025856 \&$ isnumber $=6025381$.

[8] Lab center Ltd, Proteus Introduction, http://www.labcenter.com.

[9] Ding Yanchuang; Guo Jinying; , "LED Display Screen Design and Proteus Simulation Based on Single-Chip Microcomputer," Information Engineering and Computer Science (ICIECS), vol., no., pp.1-4,25-26Dec.2010 doi:10.1109/ICIECS.2010.5677762

URL:http://ieeexplore.ieee.org/stamp/stamp.jsp?tp=\&arn umber $=5677762 \&$ isnumber $=5677633$. 